\title{
Thorough Analysis of 802.11e Star Topology Scenarios in the Presence of Hidden Nodes
}

\author{
Katarzyna Kosek ${ }^{1}$, Marek Natkaniec ${ }^{1}$, and Luca Vollero ${ }^{2}$ \\ ${ }^{1}$ AGH University of Science and Technology, Department of Telecommunications \\ al. Mickiewicza 30, 30-059 Kraków, Poland \\ ${ }^{2}$ Consorzio Interuniversitario Nazionale per l'Informatica, Laboratorio ITeM-CINI \\ Complesso Universitario Monte S.Angelo-Edificio Centri Comuni \\ Via Cinthia, 80126 Napoli \\ \{kkosek, natkaniec, vollero\}@ieee.org
}

\begin{abstract}
In this paper the authors present a simulation study of two 802.11e network scenarios. The presented analysis is not only novel but most of all crucial for understanding how a theoretically simple star topology network can be degraded by the presence of hidden nodes. The authors discuss the results obtained during the analysis of two different star topologies where the hidden node problem exists and compare them with the corresponding ones without hidden nodes. Additionally, the usefulness of the four-way handshake mechanism is argued and, furthermore, brief descriptions of the currently known solutions of the hidden node problem are given. Finally, the authors signalize the need for a better MAC protocol and provide a number of important conclusions about the 802.11e nature.
\end{abstract}

Key words: hidden nodes, QoS, IEEE 802.11e.

\section{Introduction}

Wireless networking is currently one of the most evolving technologies which importance grows constantly. Wireless devices appear almost everywhere - at homes, in companies, public places, etc. and help in everyday life. However, one of the most interesting access technologies, from the average user perspective, are ad-hoc networks. These are networks without infrastructure which do not need complicated administration and have one more undoubted advantage, i.e., they greatly facilitate Internet access. Unfortunately, wireless networks were created to deal with data exchanges and not multimedia services. Therefore, the need for QoS assurance for delay sensitive and/or bandwidth consuming services remains an interesting and unresolved issue. The nature of ad-hoc networks (i.e., constantly changing and unpredictable channel conditions, varying load, changeable device performance, different transmission and sensing ranges, mobility, hidden and exposed node problems, etc.) make it even more difficult. In this article the authors focus on the hidden node problem because it seems the most interesting from their point of view.

The purpose of simulating star topologies is simple. A good example of such a case in a real environment is a situation with a gateway $(\mathrm{GW})$ and several nodes with unidirectional antennas. The nodes need to communicate with the GW every time they 
access the Internet services. At the same time, these nodes stay hidden from each other and can hear only the GW's transmissions. Therefore, due to the fact of high popularity of the described topology, the authors found it crucial to check if $802.11 \mathrm{e}$ can assure QoS guarantees in such environments. Preliminary results of the star topologies simulations can be found in [10]. However, they are less scrupulous than the ones presented in this paper. In [10] only one configuration of a five-node star scenario was analyzed and it was not compared to any other configuration. The analysis presented in this article helps draw new and more thorough conclusions about 802.11e. Additionally, it shows similarity in the performance of the four and five node star topology which helps in formulation of general conclusions. Among many consequences of the hidden nodes presence, the most important seems the unavoidable unfairness in granting medium access and distortion of the throughput levels obtained by different priority traffic streams. The paper gives also a brief description of the known solutions of the hidden node problem and argues the usefulness of the most commonly used four-way handshake.

The remainder of this paper is organized as follows. Section 2 contains the state of the art. In Section 3 the simulation scenarios description can be found. Section 4 gives conclusions on the obtained results which can be found in Appendix. The concluding remarks can be found in Section 5.

\section{State of the Art}

\section{The IEEE 802.11e Standard}

Quality of Services support in wireless networks is provided by the IEEE $802.11 \mathrm{e}$ standard [1]. The standard defines the Hybrid Coordination Function (HCF), which includes the HCF Controlled Channel Access (HCCA) and the HCF contention-based channel access, also known as the Enhanced Distributed Channel Access (EDCA). HCCA and EDCA are interoperable channel access mechanisms. HCCA is based on polling, while EDCA is based on a slotted and highly parametric CSMA/CA protocol. Both mechanisms distribute Transmission Opportunities (TXOPs), in which nodes are allowed to transmit one or more data frames.

EDCA defines the concept of Access Category $(A C)$. Each node may use up to four $A C$ s which represent four priority levels for data transmission. The standard names these levels as background (BK), best effort (BE), video (Vi) and voice (Vo). Each AC implements a slotted CSMA/CA algorithm with its own parameters and competes with other $A C$ s in order to obtain TXOPs. When it obtains a TXOP, it can transfer at least the first frame waiting in its transmission queue. Moreover, the AC can transmit more frames if allowed. Specifically, each $A C$ has a maximum channel occupancy time, called $T X O P_{\text {limit }}$ which is a configurable QoS parameter. If its $T X O P_{\text {limit }}$ is equal to zero, the $A C$ is allowed to transmit only the one frame for each TXOP it gains. When the $T X O P_{\text {limit }}$ is greater than zero, the $A C$ is allowed to transmit as long as the total channel occupancy time is less or equal than the $T X O P_{\text {limit. }}$. An idle $A C$ starts competing for a TXOP upon the arrival of a new frame in its queue. If the frame arrives and no more $A C$ s are active in the same node, the $A C$ checks the wireless medium if it is idle or busy. If the channel is idle, the $A C$ ensures that it remains idle for a fixed interval of time, i.e., the Arbitration Inter-Frame Space (AIFS $[A C])$, which is 
another configurable QoS parameter. As soon as the AIFS has expired, the $A C$ is allowed to transmit. If the transmission is successful, the receiving node transmits back to the sender a special acknowledgement (ACK) frame, acknowledging the successful transmission. The $A C$ that has obtained the TXOP handles the transmission of all the frames waiting in its queue until its $T X O P_{\text {limit }}$ has been consumed. If the transmission is unsuccessful, the $A C$ enters the Backoff process. This process is also used when the channel is sensed busy during the first AIFS, when another $A C$ in the same node is busy or the last $T X O P_{\text {limit }}$ of the $A C$ is too close in time. As soon as the Backoff process is started, the $A C$ updates an internal variable, called Backoff Timer $(B T)$. When updating, the value of the $B T$ is extracted randomly in the set: $\left\{0,1, \ldots, \min \left(C W \max [A C], 2^{k} \cdot C W \min [A C]\right)\right\}$, where $C W \min [A C]$ and $C W \max [A C]$ are the minimum and the maximum Contention Window and $k$ is the number of collisions occurred to the current frame. $C W \min$ and $C W \max$ are also configurable QoS parameters. An $A C$ with $B T$ equal to zero is allowed to attempt a transmission in the first slot time following an idle AIFS or an empty slot time. The $B T$ is decremented in each slot time following an AIFS or an empty slot time.

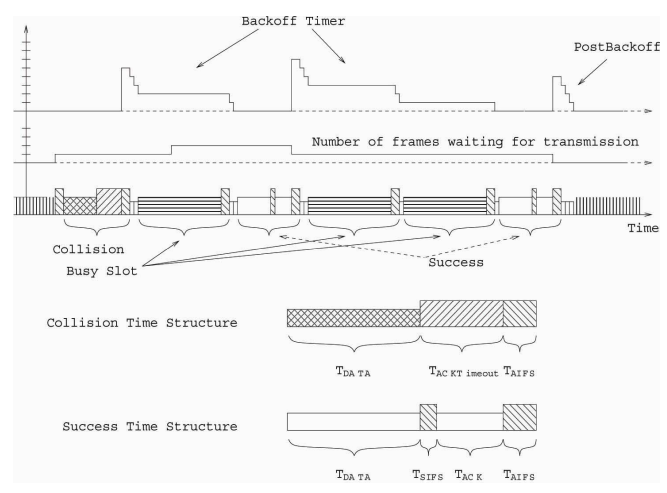

Fig. 1. AC channel access time-sequence

An example of the EDCA access mechanism is shown in Fig. 1 (with $T X O P_{\text {limit }}$ equal to zero). If the queue of the $A C$ is empty after its last successful transmission, the standard mandates the updating of the $B T$. This Post-Backoff is executed as the normal Backoff process. If this queue is empty also when the BT has expired, the $B T$ remains equal to zero until a new frame arrives. If a new frame arrives before the $B T$ has expired, it is served using the Backoff process, inheriting the current $B T$ value of the $A C$.

Due to the fact that EDCA is the basic IEEE 802.11e mode implemented in real devices the authors find it crucial to check if it can assure QoS guarantees in different star topologies. TXOP $P_{\text {limit }}$ different from zero was not analyzed during the simulations they performed.

\section{The Hidden Node Problem}

One of the meaningful disadvantages of wireless networks is that even though the possible PHY Layer rates are satisfactorily high the MAC Layer is not able to use the 
whole bandwidth. There are two main reasons of such performance. First of all, the current MAC proposals are suboptimal. Secondly, node starvation is unavoidable as long as hidden nodes are present within wireless networks. Hidden nodes may appear due to the half-duplex nature of the wireless devices, i.e., when two nodes are out of range of each other they are unable to hear their transmissions. Therefore, if they start their data transmissions simultaneously a physical collision must happen.

\section{Solutions of the Hidden Node Problem}

The most known solution being used to minimize the destructive effects of the hidden nodes is the four-way handshake. The mechanism uses four different types of frames, i.e., Request to Send (RTS), Clear to Send (CTS), Data (DATA) and Acknowledgement $(\mathrm{ACK})$ which are exchanged during the process of granting the medium access. Unfortunately, this solution has several disadvantages among which only the most important ones are given next. Firstly, it is optional and, therefore, not always used. Secondly, this method minimizes the problem of hidden nodes only when the network is of a single-hop type. Thirdly, this mechanism consumes bandwidth even if no hidden nodes appear within a network. Finally, due to the exchange of the additional RTS/CTS frames, the mechanism is unsuitable for delay-sensitive traffic.

An important improvement to the four-way handshake is Multiple Access with Collision Avoidance for Wireless (MACAW, [5]) where five different types of frames are exchanged, i.e., RTS, CTS, Data Sending (DS), DATA and ACK. Additionally, to increase the per-node fairness MACAW involves a Request to RTS (RRTS) control frame. The biggest weakness of MACAW is the unsolved exposed node problem and furthermore, the increased signaling overhead. Therefore, in contrast to the senderinitiated handshake mechanisms, a family of receiver-initiated mechanisms has been proposed. In these types of channel granting solutions, the receiver must poll its neighbors in order to check if they have packets destined to it. The most known is Multiple Access Collision Avoidance By Invitation (MACA-BI, [6]) where a threeway handshake mechanism is invoked for every frame transmission, i.e., CTS/DATA/ACK. However, this mechanism is unsuitable for ad-hoc networks where polling a station without packets to be sent is a waste of time. Due to the weaknesses of previous mechanisms, hybrid solutions have appeared (e.g., [7]) which take advantages of both receiver and sender-initiated channel access methods. They assure better fairness and decrease end-to-end delay. However, they cannot guarantee QoS for delay sensitive traffic and were tested only in 802.11 environments. There also exists a family of protocols involving busy tone signals. The two most known solutions are Busy Tone Multiple Access (BTMA, [8]) and Dual Busy Tone Multiple Access (DBTMA, [9]). BTMA is dedicated only to networks with infrastructure. DBMA uses two busy tone signals and two sub-channels (i.e., communication and control) but it does not pay enough attention to the possible interference on the control channel and does not involve ACKs which seems illogical in the case of unreliable wireless links. One other solution is Floor Acquisition Multiple Access with Non-persistent Carrier Sensing (FAMA-NCS, [11]). It takes advantage of using long CTS frames which aim is to prevent any contending transmissions within the receiver's range. Unfortunately, this scheme requires all nodes to hear the interference what makes the mechanism inefficient in case of short DATA frames. 

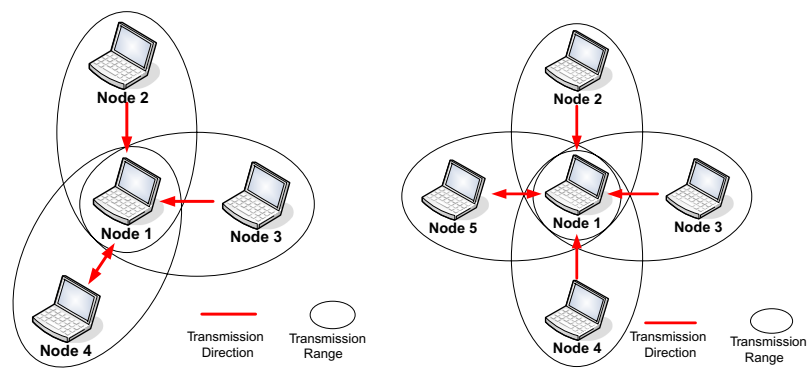

Fig. 2. Four-node (a) and five-node (b) star topology network

assumption is made in order to avoid ineffective transmissions of small frames and, primarily, to compare the four EDCA queues under similar conditions. The analysis was performed with basic channel access (DATA/ACK) and with the four-way handshake mechanism (RTS/CTS/DATA/ACK). In the presented figures showing throughput and frame loss the errors do not exceed $\pm 2 \%$ assuming $95 \%$ confidence intervals.

\section{Simulation Results}

Three separate configurations were analyzed in order to have enough data for general conclusions. The results obtained for the four-node star were compared to the ones obtained for the five-node star. They appeared quite similar, i.e., not only the general conclusions but also the specific ones (e.g., the relations of the throughput levels to the number of DATA collisions (COL), interface queue (IFQ) drops, address resolution protocol (ARP) drops, and MAC retransmissions (RET) drops) were the same. Only the curve levels were different (i.e., smaller overall throughput and higher number of the lost frames were observed for the five-node star). Since this similarity and for the clarity of presentation, only the five-node configurations are presented in detail. However, the general conclusions form Section 5 account also for the results obtained in the four-node configurations. Eventually, due to the lack of space, only the overall frame loss curves are presented in the paper. They represent an aggregated number of IFQ, RET and ARP drops.

\section{A. First Configuration}

First configuration results are shown in Fig. 3 and Fig. 6. In this setup the number of low priority streams, 4 , is higher than that of high priority ones, 1. Fig. 3-(a) shows that the order of the throughput levels is slightly distorted. For the hidden nodes, VI has the highest throughput while BE and BK have lower. However, the unhidden N1, sending BK traffic, achieves significantly higher throughput than the hidden nodes sending BK and BE. Moreover, the throughput of N1 is almost the same as that of N4 (which sends VI priority traffic) and the throughput levels of the low priority streams (i.e., for N2, N3, and N5) are unacceptably low. Furthermore, when the four-way handshake mechanism is enabled the fairness is slightly better but it is still not ideal 
(c.f., the dashed lines in Fig. 3). On the basis of these observations the following question can be formulated:

Why is the throughput: $t h_{N 4}>t h_{N 1}>t h_{N 2} \cong t h_{N 3} \cong t h_{N 5}$ ?

When the four-way handshake is enabled, N4 has almost three times more COLs and two times less RET drops than other hidden nodes and between 1.2 and 1.4 times fewer IFQ drops than any other node. The number of COLs is about 32 times lower than RET drops and, therefore, it can be concluded that the predominant number of frames are received by the destination without the need of MAC retransmissions. N1 has the smallest number of COLs and RETs, however, it has also more IFQ drops than N4. Therefore, the explanation why N4 has generally higher throughput than the unhidden N1 is due to its priority traffic class. The high number of collisions experienced by $\mathrm{N} 4$ is also caused by its data traffic priority because the higher the priority, the higher the probability that the node will try to gain access to the medium. It can collide more often with other nodes but on the other hand it can also have fewer frames in its MAC priority queues. N1 experiences hardly any collisions because it is the only unhidden node and can hear other transmissions practically all the time. However, when N1 transmits without colliding, it completely captures the channel avoiding any interference by other nodes. The overall summarization of frame loss shows that the number of lost frames for N4 is lower than the ones observed for other nodes, which stays in line with the throughput level it achieved. With RTS/CTS disabled the overall frame loss situation is almost similar to the previous one because basically only the curves' levels change. The trend of the curves representing the number of IFQs for N1 is a bit higher and for N4 is a bit lower. The number of COLs is reduced practically to zero for all nodes. Also the number of RET drops changes significantly and for all hidden nodes is almost two times lower than with RTS/CTS disabled. Therefore, the throughput levels changed accordingly (c.f., the dashed lines in Fig. 3)

Another experiment was performed to increase the hidden nodes' carrier sensing range so as to not make them hidden any more. The obtained results are presented in Fig.6. In such a configuration the medium access was fair. However, it is also noticeable that enabling RTS/CTS causes a decrease in the overall throughput (mostly due to the increase in the signaling overhead and low sending rate of RTS/CTS frames which is $2 \mathrm{Mb} / \mathrm{s}$ ) which results in worse overall network performance. Therefore, it seems reasonable to disable four-way handshake in star environments where the problem of hidden nodes does not exist. Nodes with BK priorities have the highest number of lost frames which is lower than the number observed for BE which is in turn lower than the number for VI. With RTS/CTS enabled the number of frames lost for all priorities is higher than with RTS/CTS disabled. The most important impact on frame loss comes from IFQ drops (the number of which is incomparably higher than the number of ARP or MAC retransmission drops). Therefore, practically only the IEEE 802.11e mechanism has impact on network performance.

\section{B. Second Configuration}

The second configuration is presented in Fig. 4 and Fig. 7. The most important change with respect to the previous configuration is that N1 has VO priority instead of BK and, additionally, there are more high priority streams, 3, than low priority ones, 2 . 
Important conclusions from the results shown in Fig. 4 are as follows: N1 gains the medium access more often than any other node both with enabled and disabled RTS/CTS. When the four-way handshake mechanism is enabled the fairness of granting medium access is better, i.e., hidden nodes sending VI and VO have better throughput than the ones sending BK. The throughput of N5, with disabled RTS/CTS, equals zero. With a smaller offered load (up to $0.4 \mathrm{MB} / \mathrm{s}$ ), the throughput of $\mathrm{N} 2$ and $\mathrm{N} 3$ is higher than the throughput of N4, even though they send lower priority traffic. The dominance of $\mathrm{N} 1$ over hidden nodes is higher than it was presented in the second case, therefore, the unanswered questions are:

\section{Why is $t h_{N 1} \gg t h_{N 2} \cong t h_{N 3} \cong t h_{N 4} \geq t h_{N 5}$ with RTS/CTS disabled?}

N5 experiences the greatest number of COLs which is, however, practically equal to the number observed for N4. Also the number of RET drops is highest for these two nodes. The number of COLs for N2 and N3 are practically equal and lower than the ones observed for N5 and N4. Additionally, they experience lower number of RET drops than N5 and N4 but visibly more than N1. In general, the number of frame drops observed for hidden nodes can be explained by the comparable priorities of the traffic carried by N5 and N4 and by N2 and N3. Higher priority traffic can be send more often experiencing more COLs, as it was already described in the previous configuration. N1 has almost no COLs and, therefore, also the number of RET drops falls to zero. Such performance is caused by the unhidden placement of N1. The number of IFQ drops is highest for N2 and N3, a bit lower for N4 and N5 and lowest for N1, while, the overall frame loss is almost equal for all hidden nodes if only the offered load is higher than $0.4 \mathrm{Mb} / \mathrm{s}$ and much higher than the one observed for N1.

\section{Why is $t h_{N 1} \gg t h_{N 5}>t h_{N 4}>t h_{N 3} \geq t h_{N 2}$ with RTS/CTS enabled?}

The number of COLs decreased almost to zero for all nodes. The number of RET drops was lowest for N1 (equal to 0), a bit higher for N2 and N3 and highest for N4 and N5. The number of IFQ drops was practically equal for N2, N3 and was slightly higher than the ones observed for N4 and N5 and much higher than the one observed for N1. These numbers can be explained by the priorities of the traffic carried by the particular hidden nodes and by the placement of the unhidden N1. The overall frame loss was highest for N2 and N3, lower for N4, a bit more lower for N5 and lowest for $\mathrm{N} 1$ - this determines the obtained throughput.

Fig. 7 shows the ideal case, i.e., the environment in which no hidden nodes are present. Frame loss levels obtained by the competing stations are in the correct order which is in line with the obtained throughput. Once again enabling the RTS/CTS exchange causes a meaningful decrease in the overall network throughput and increase in the overall frame loss for each priority. In both cases, IFQ drops have the strongest impact on the achieved results.

\section{Third Configuration}

The third configuration is shown in Fig. 5 and Fig.8. There are slightly more low priority streams, 3, than high priority ones, 2 . The list of the most important conclusions based on the results presented in Fig. 5 is as follows: N1 had lower traffic priority than in the previous case which resulted in better fairness in granting medium access when the four-way handshake was enabled. N1 tried to send data more seldom, 
what is a result of the high $C W \min$ and $C W \max$ values connected with the $\mathrm{BK}$ traffic. Unfortunately, once more with disabled RTS/CTS the nodes with P0 and P1 priorities did not get enough bandwidth when the hidden nodes were present in the network. Up to the value of the overall traffic load of $0.3 \mathrm{MB} / \mathrm{s}, \mathrm{BE}$ and $\mathrm{BK}$ streams took advantage over VI and, up to $0.4 \mathrm{MB} / \mathrm{s}$, over VO. Additionally, the throughput of N5 with disabled RTS/CTS once again equals zero. The change in fairness is noticeable mostly when the four-way handshake is enabled, i.e., hidden nodes sending VI and VO traffic have better throughput than the ones sending BE and BK traffic. However, in comparison to configuration 1, the VO traffic levels are severely distorted. The explanation of such performance is given next.

\section{Why is $t h_{N 1} \gg t h_{N 3} \cong t h_{N 4} \cong t h_{N 5} \geq t h_{N 2}$ with RTS/CTS disabled?}

The number of COLs was highest for N2 and N3, lower for N4 and N5 and lowest for $\mathrm{N} 1$ (practically equal to zero). The number of RET drops was highest for N3, lower for N2, a bit more lower for N4 and N5 and lowest for N1 (equal to zero). These numbers can be explained by the traffic priorities and the unhidden position of $\mathrm{N} 1$. The number of IFQ drops was highest for N4 and N5, lower for N3 and N2 and lowest for N1. The overall frame loss was smallest for N1 and practically equal to the hidden nodes which explains the throughput levels obtained.

\section{Why is $t h_{N 1}>t h_{N 2}>t h_{N 3}>t h_{N 4} \cong t h_{N 5}$ with RTS/CTS enabled?}

The number of COLs dropped practically to zero for all nodes. The number of RET drops was highest for N3, lower for N2, another bit lower for N4 and N5 and lowest for N1 (equal to zero). The order of IFQ drops numbers was smallest for N1, higher for N2, another bit higher for N3 and highest for N4 and N5. However, the overall frame loss was highest for N4 and N5, smaller for N3, smaller for N2 and smallest for N1. Therefore, it can be assumed that for the hidden nodes it was $802.11 \mathrm{e}$ mechanism which mostly determined their performance. Obviously, the dominant role of N1 was caused by its unhidden placement. Furthermore, in comparison to configuration 1, the placement of N1 was even more important as the traffic sent by hidden nodes was generated and collided more often. It was caused by the fact that one of the hidden nodes was sending $\mathrm{P} 0$ instead of $\mathrm{P} 2$.

Fig. 8 presents the ideal environment, e.g. the same configuration without hidden nodes. The curves representing throughput have the expected shapes, i.e., better priority traffic has higher throughput than the low priority traffic, as well as, the same priority streams have the same throughput values. Frame loss curves have also the expected order and they impact on the throughput obtained by different nodes. Enabling RTS/CTS exchange causes decrease in the overall throughput and increase in the number of lost frames. With both enabled and disabled four-way handshake the main impact on the nodes' throughput comes from IFQ drops.

\section{Conclusions and Future Work}

The paper analyzed the impact of EDCA parameters in WLAN configurations where hidden nodes are present and compared them with configurations where the problem of hidden nodes did not exist. In the presented scenarios the imperfect channel sensing is a major cause of the asymmetry in resource allocation. Additionally, novel 
simulation analysis showed that current EDCA cannot be used to counterbalance this effect alone if hidden nodes are present within a particular wireless network. As shown in the figures with RTS/CTS disabled, the higher the priority of traffic is carried by the hidden stations the worse the throughput level they achieve. Furthermore, with RTS/CTS enabled, the problem of severe unfairness still exists. In both cases, node starvation is much worse when the unhidden station transmits high priority traffic. The analysis showed also that similar configurations behave similar, i.e., the order of throughput levels obtained by different nodes for a five- and a four-node star is exactly the same regardless of the traffic priority the unhidden station transmits. This suggests that common strategies for avoiding the hidden nodes' impact on EDCA may exist. The authors' future works will be mainly focused on the definition of such strategies and on embedding them in ad-hoc protocols. Additionally, the authors plan to analyze different scenarios with a varied order of traffic priorities. They will analyze cases with a smaller and a higher number of hidden nodes in order to formulate even more general conclusions which will help in defining a new MAC protocol.

\section{Acknowledgement}

This work has been carried out under the EU founded NoE CONTENT project no. 038423. The authors would like to thank OPNET Technologies, Inc. for supporting this research by providing the OPNET Modeler software.

\section{References}

[1] I.S.: 802.11e 2005 IEEE 802.11 WG Part 11: Wireless LAN Medium Access Control (MAC) and Physical Layer (PHY) Specifications Amendment 8 (2005)

[2] IEEE 802.11b: Higher-speed PHY extension in the $2.4 \mathrm{GHz}$ band (1999)

[3] TKN EDCA 802.11e extension to the network simulator ns2. [Online] http: / /www.tkn.tu-berlin.de/research/802.11e_ns2

[4] OPNET. [Online] http: / / www. opnet.com

[5] Bharghavan, V., Demers, A., Shenker, S., Zhang, L.: MACAW: A Media Access Protocol for Wireless LAN's. In: ACM SIGCOMM 1994, UK (September 1994)

[6] Talucci, F., Gerla, M., Fratta, L.: MACA-BI (MACA by invitation) - A Receiver Oriented Access Protocol for Wireless Multihop Networks. In: Proc. IEEE PIMRC 1997, Finland (September 1997)

[7] Wang, Y., Garcia-Luna-Aceves, J.J.: A New Hybrid Channel Access Scheme for Ad Hoc Networks. In: MedHocNet 2002, Italy (September 2002)

[8] Tobagi, F.A., Kleinrock, L.: Packet switching in radio channels: Part II-The hidden terminal problem in carrier sense multiple-access and the busy-tone solution. In: IEEE Transactions on Communications (December 1975)

[9] Haas, Z.J., Deng, J.: Dual Busy Tone Multiple Access (DBTMA) - A Multiple Access Control for Ad Hoc Networks. IEEE Transactions on Communications (June 2002)

[10] Kosek, K., Natkaniec, M., Vollero, L., Pach, A.R.: Performance Analysis of 802.11e Networks with Hidden Nodes in a Star Topology. In: CCNC 2008, USA (January 2008, short paper)

[11] Fullmer, C.L., Garcia-Luna-Aceves, J.J.: Solutions to Hidden Terminal Problems in Wireless Networks. In: Proc. ACM SIGCOMM 1997, France (September 1997) 


\section{Appendix}
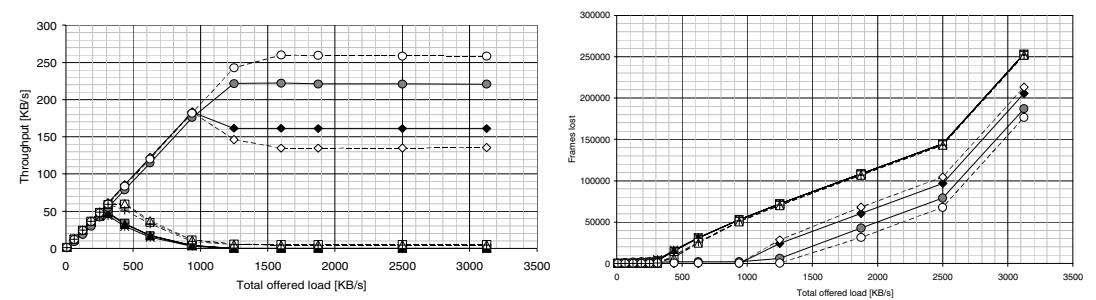

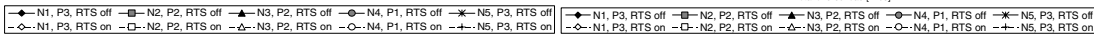

Fig. 3. Configuration 1 - network with hidden nodes: (a) throughput (b) frame loss
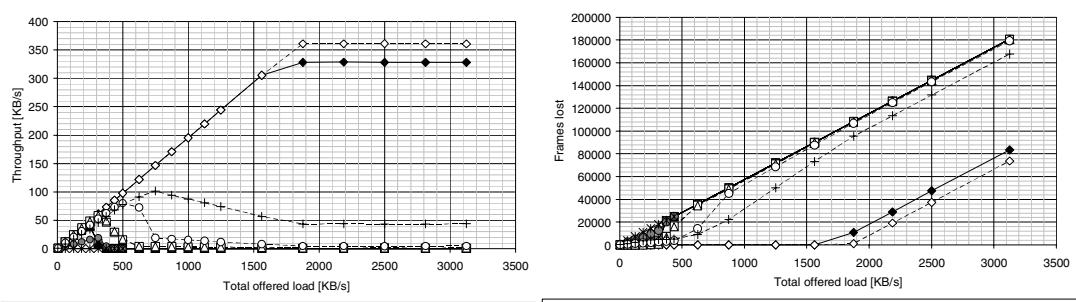

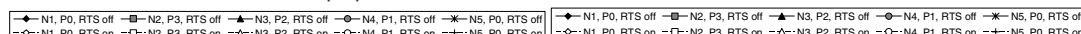

Fig. 4. Configuration 2 - network with hidden nodes: (a) throughput (b) frame loss
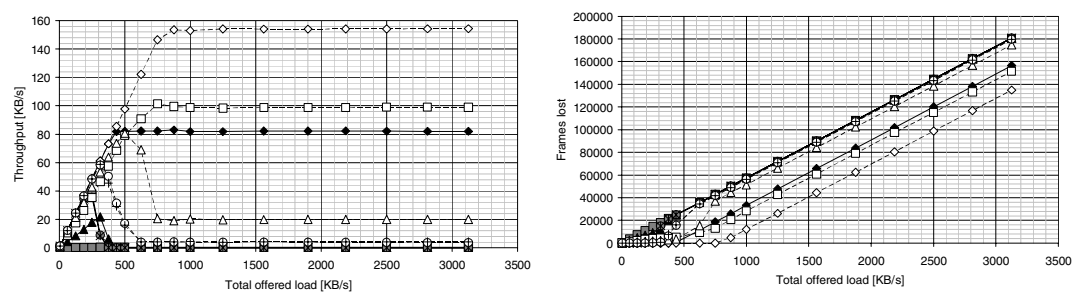

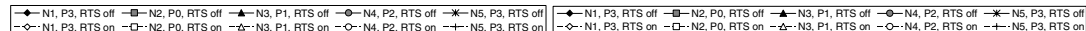

Fig. 5. Configuration 3 - network with hidden nodes: (a) throughput (b) frame loss
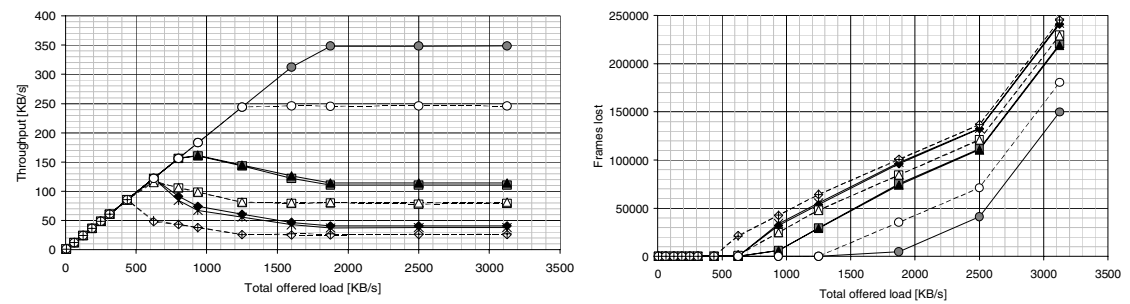

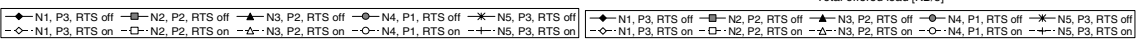

Fig. 6. Configuration 1 - network without hidden nodes: (a) throughput (b) frame loss 

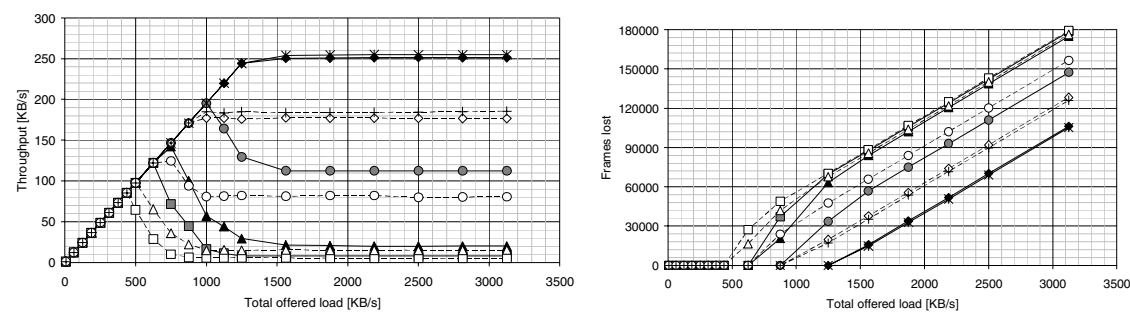

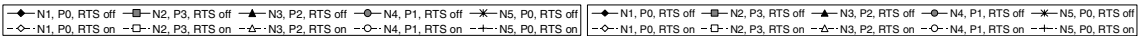

Fig. 7. Configuration 2 - network without hidden nodes: (a) throughput (b) frame loss
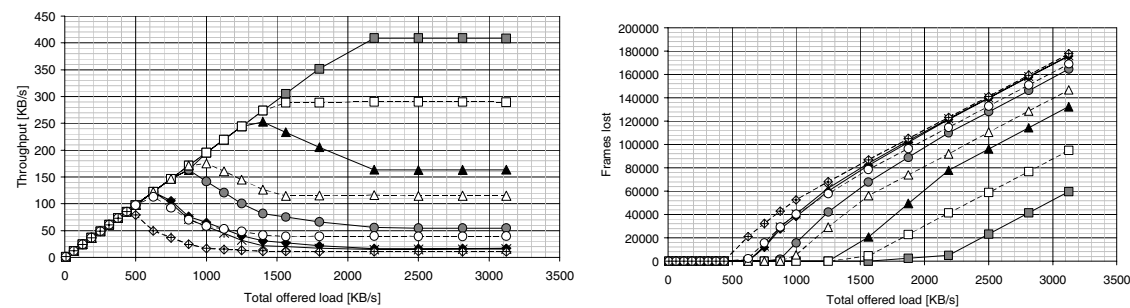

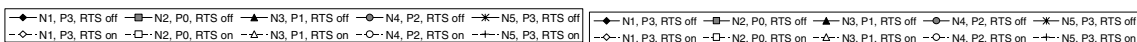

Fig. 8. Configuration 3 - network without hidden nodes: (a) throughput (b) frame loss 